\title{
Types I and II Keratin Intermediate Filaments
}

\author{
Justin T. Jacob, ${ }^{1}$ Pierre A. Coulombe, ${ }^{1,2}$ Raymond Kwan, ${ }^{3}$ \\ and M. Bishr Omary ${ }^{3,4}$
}

\begin{abstract}
${ }^{1}$ Department of Biochemistry and Molecular Biology, Bloomberg School of Public Health, Johns Hopkins University, Baltimore, Maryland 21205

${ }^{2}$ Departments of Biological Chemistry, Dermatology, and Oncology, School of Medicine, and Sidney Kimmel Comprehensive Cancer Center, Johns Hopkins University, Baltimore, Maryland 21205

${ }^{3}$ Departments of Molecular \& Integrative Physiology and Medicine, University of Michigan, Ann Arbor, Michigan 48109

${ }^{4}$ VA Ann Arbor Health Care System, Ann Arbor, Michigan 48105

Correspondence: coulombe@jhsph.edu
\end{abstract}

\section{SUMMARY}

Keratins - types I and II-are the intermediate-filament-forming proteins expressed in epithelial cells. They are encoded by 54 evolutionarily conserved genes (28 type I, 26 type II) and regulated in a pairwise and tissue type-, differentiation-, and context-dependent manner. Here, we review how keratins serve multiple homeostatic and stress-triggered mechanical and nonmechanical functions, including maintenance of cellular integrity, regulation of cell growth and migration, and protection from apoptosis. These functions are tightly regulated by posttranslational modifications and keratin-associated proteins. Genetically determined alterations in keratin-coding sequences underlie highly penetrant and rare disorders whose pathophysiology reflects cell fragility or altered tissue homeostasis. Furthermore, keratin mutation or misregulation represents risk factors or genetic modifiers for several additional acute and chronic diseases.

\section{Outline}

\section{Introduction}

2 Introduction to keratin IF genes and proteins

3 Assembly, properties, and organization of keratin filaments in epithelial cells

4 Keratin filaments are essential for the maintenance of cellular integrity in the face of stress $-\mathrm{A}$ role that is defective in epithelial fragility disorders

5 Emergence of noncanonical functions for keratin proteins and their implication in disease
6 An interplay between keratin and cytoarchitecture involves prominent interactions with the plakin family

7 Keratin regulation by posttranslational modifications

8 Conclusion

References

Editors: Thomas D. Pollard and Robert D. Goldman

Additional Perspectives on The Cytoskeleton available at www.cshperspectives.org

Copyright $\odot 2018$ Cold Spring Harbor Laboratory Press; all rights reserved; doi: 10.1101/cshperspect.a018275 


\section{INTRODUCTION}

Intermediate filaments (IFs) are one of three major cytoskeletal filament assemblies in higher eukaryotes. The designation "intermediate filaments" conveys that their diameter, $\sim 10 \mathrm{~nm}$, is intermediate between those of actin microfilaments $(\sim 6-8 \mathrm{~nm})$ and microtubules $(\sim 25 \mathrm{~nm})$ (Ishikawa et al. 1968). Proteins belonging to this large superfamily, comprising approximately 70 conserved genes, show substantial amino acid sequence diversity, with some sharing as little as $20 \%$ identity (Szeverenyi et al. 2008; Strnad et al. 2011). Regardless of this heterogeneity, each member of the IF family shares a tripartite protein substructure, with a family-defining, centrally located $\alpha$-helical rod domain, and readily self-assembles to form $10-\mathrm{nm}$ filaments on its own or, more commonly, in partnership with other IF proteins (Fig. 1A-C) (Herrmann and Aebi 2004; for review, see Herrmann and Aebi 2016). The central $\alpha$ helical domain, denoted as the "rod," is flanked by two highly variable, nonhelical domains at the amino and carboxyl termini called the "head" and "tail," respectively (Fig. 1A). Based on criteria including gene substructure, primary structure of the protein products, and tissue distribution, the 70-plus known IF genes and proteins can be readily partitioned into six major classes or types (Kim and Coulombe 2007; Szeverenyi et al. 2008). Keratins (formerly referred to as "cytokeratins") represent types I and II IF genes and proteins and are expressed primarily in epithelial cells (Fig. 1D,E). Here, we review the main properties of keratin genes and proteins, their regulation and functions in vivo, and their involvement in a broad array of human diseases.

\section{INTRODUCTION TO KERATIN IF GENES AND PROTEINS}

Keratin proteins $\left(M_{r}: 40-70 \mathrm{kDa}\right)$ are the predominant subtype of IFs in all epithelia and, given a total of 54 conserved functional genes and proteins, represent nearly three-quarters of the entire IF superfamily in mammals (Szeverenyi et al. 2008). Keratins comprise two types of IF sequences or families: type I, which number 28 , and type II, which number 26 (Fig. 2A). A new nomenclature was devised in 2006 to accommodate the identification of many new keratin genes, brought about in part by the sequencing of the human and mouse genomes (Hesse et al. 2004; Schweizer et al. 2006). Types I and II keratins are very well demarcated from a perspective of gene substructure as well as of protein sequence homology over their central $\alpha$-helical rod domain. They also can be easily resolved by two-dimensional gel electrophoresis, given that type I keratin proteins tend to be smaller $(40-56.5 \mathrm{kDa})$ and acidic in overall charge (pI 4.5-6.0), whereas type II
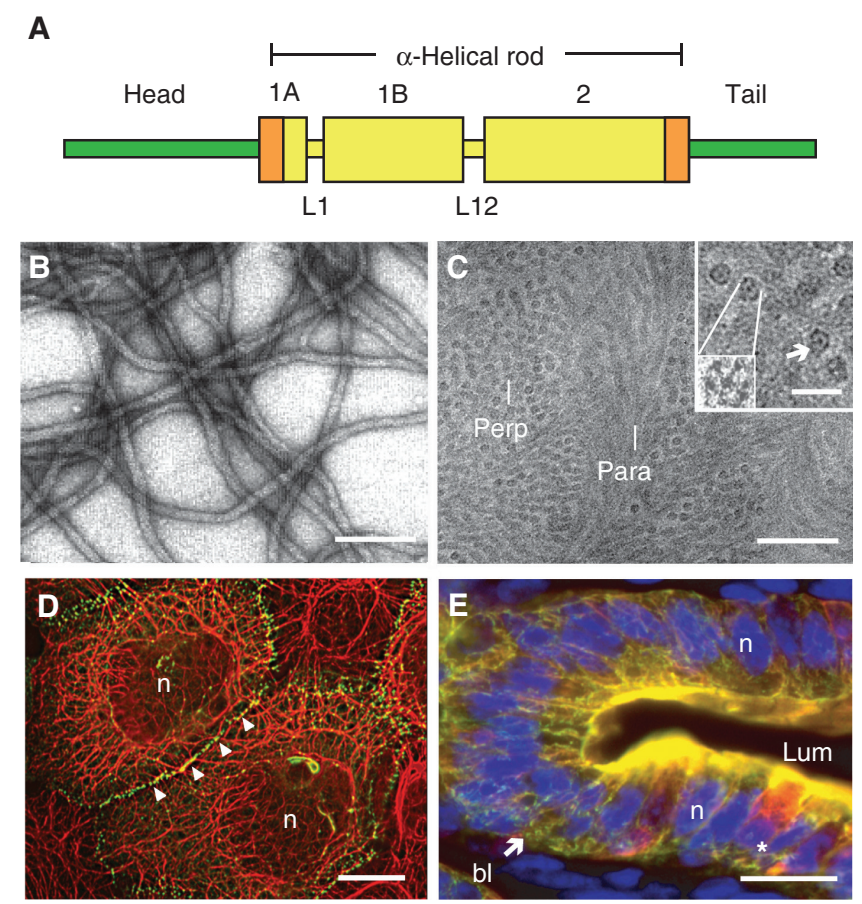

Figure 1. Introduction to keratin intermediate filaments (IFs). (A) Schematic representation of the tripartite domain structure shared by all keratin and other IF proteins. A central $\alpha$-helical "rod" domain acts as the major determinant of self-assembly and is flanked by nonhelical "head" and "tail" domains at the amino and carboxyl termini, respectively. Within this 310-amino-acidlong rod domain, the heptad-repeat-containing segments (e.g., coils 1A, 1B, and 2) are interrupted by linker sequences at two conserved locations (e.g., L1 and L12). Rod domain boundaries consist of highly conserved 15-20-amino-acid regions (shown in orange) that are crucial for polymerization and frequently mutated in human disease (see www.interfil.org). (B) Visualization of filaments, reconstituted in vitro from purified human K5 and K14 proteins, by negative staining and electron microscopy. Scale bar, $100 \mathrm{~nm}$. (C) Visualization of native keratin IFs in the stratum corneum layer of human epidermis using cryo-transmission electron microscopy on a fully hydrated, vitreous tissue specimen (Norlén and Al-Amoudi 2004). Bundles of tightly packed keratin IFs run parallel (para) or perpendicular (perp) to the sectioning plane. Scale bar, $50 \mathrm{~nm}$. (Inset) Detailed views of filaments in cross section, shown at two magnifications. As many as seven subfibrils, including a centrally located one, can be seen. Scale bar, $20 \mathrm{~nm}$. (D) Skin epidermal keratinocytes in culture. Dual-immunofluorescence labeling for keratin (red signal) and desmoplakin (green signal), a desmosome component. Keratin IFs are organized in a network that spans the whole cytoplasm and are attached to desmosomes, which are points of adhesion at cell-cell contacts. $n$, Nucleus. Scale bar, $20 \mu \mathrm{m}$. (E) Gut epithelial wall in cross section, emphasizing the epithelium. This fresh-frozen specimen was triple-labeled for K8/K18 (red signal), K19 (green signal), and nuclei (blue signal). Note the concentration of staining at the apical pole of enterocytes. The star denotes a goblet cell, which also features a prominent K8/K18 network in the cytoplasm. bl, Basal lamina; lum, lumen; $n$, nucleus. Scale bar, $20 \mu \mathrm{m}$. (Reprinted, with permission, from Kim and Coulombe 2007; C, originally adapted from Norlén and Al-Amoudi 2004, with permission from Elsevier.) 
A
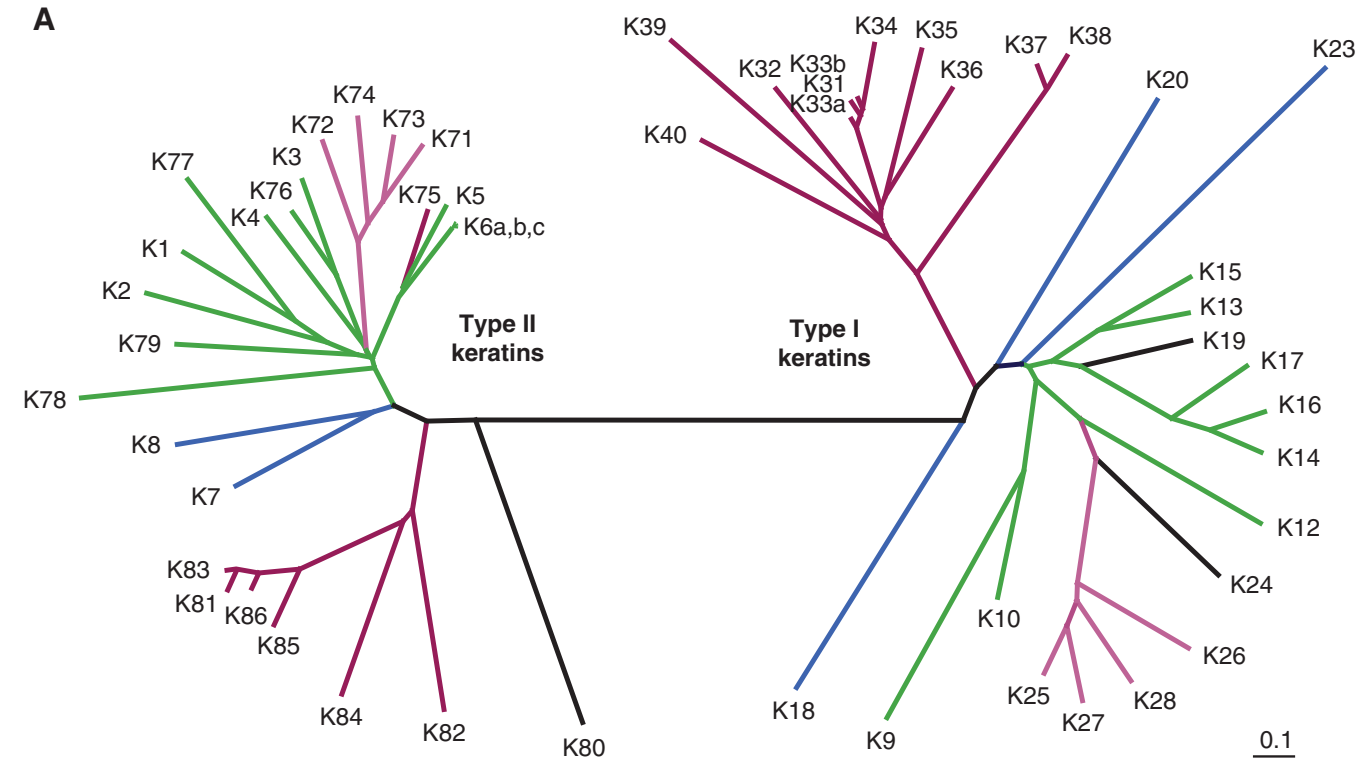

B

Type I keratin gene cluster - Human chromosome 17q21.2

centromere ... KRT222P KRT24 KRT223P KRT25 KRT26 KRT27 KRT28 KRT10 KRT12 KRT20 KRT23 KRT39 KRT40 KRT33A $\quad$ KRT33B KRT34 KRT31 KRT41P KRT37 KRT38 KRT221P KRT32 KRT35 KRT36 KRT13 KRT15 KRT19 KRT9 KRT14 KRT16 KRT17 KRT42P ... telomere

Type II keratin gene cluster - Human chromosome 12q13.13

centromere ... KRT80 KRT7 KRT121P KRT122P $\quad$ KRT81 KRT86 KRT23 $\quad$ KRT123P KRT85 KRT84 KRT82 KRT124P KRT75 KRT6B KRT6C KRT6A KRT5 KRT71 KRT74 KRT72 KRT73 KRT125P KRT2 KRT1 KRT77 KRT126P KRT127P KRT128P KRT76 KRT3 KRT4 KRT79 KRT78 KRT8 KRT18 ... telomere

Figure 2. Phylogenic tree of human keratins. (A) Comparison of the primary structure of human keratins using the publicly available ClustalW and TreeView softwares. Sequence relatedness is inversely correlated with the length of the lines connecting the various sequences, as well as the number and position of branchpoints. This comparison makes use of the sequences from the head and central rod domain for each keratin. Two major branches are seen in this tree, corresponding exactly to the known partitioning of keratin genes into types I and II sequences. Beyond this dichotomy, each subtype is further segregated into major subgroupings. $(B)$ Location and organization of genes encoding types I and II keratins in the human genome. All functional type I keratin genes, except Krt18, are clustered on the long arm $(q)$ of human chromosome 17, whereas all functional type II keratin genes are located on the long arm of chromosome 12. Krt18, a type I gene, is located at the telomeric (Tel) boundary of the type II gene cluster. The suffix $P$ identifies keratin pseudogenes. As highlighted by the color code used in frames $A$ and $B$, individual type I and type II keratin genes belonging to the same subgroup, based on the primary structure of their protein products, tend to be clustered in the genome. Moreover, highly homologous keratin proteins (e.g., K5 and K6 paralogs; also, K14, $\mathrm{K} 16$, and K17) are often encoded by neighboring genes, pointing to the key role of gene duplication in generating keratin diversity. These features of the keratin family are virtually identical in mouse (not shown). (Adapted from Coulombe et al. 2013.)

keratins are larger $(50-70 \mathrm{kDa})$ and basic-neutral in charge (pI 6.5-8.5) (Moll et al. 1982). Types I and II keratins are strictly interdependent for assembly into 10 -nm filaments, initially forming coiled-coil heterodimers at the very first stage of the process (Kim and Coulombe 2007). Reflecting, in part, this requirement, types I and II keratin genes are regulated in a pairwise fashion in epithelia (Sun et al. 1983; Fuchs 1995). Furthermore, specific pairings of keratin genes and proteins are differentially regulated depending on (1) the type of epithelial tissue (e.g., hard vs. soft, simple vs. stratified); (2) the differentiation programs being executed (e.g., epidermis vs. cornea, less-differentiated basal vs. more-differentiated suprabasal cells); and (3) biological context (e.g., normal homeostasis vs. tissue repair or disease) (Coulombe and Omary 2002; Szeverenyi et al. 2008). The significance of this regulation is not understood but 
underlies the broad use of keratins as markers in pathology laboratories worldwide to assess the tissue origin of cancers, which has therapeutic implications (Moll et al. 2008). Some cell types feature a very simple keratin composition-such is the case for adult liver hepatocytes, which only express the type II keratin 8 (K8) and type I keratin 18 (K18). Other epithelial cells (e.g., terminally differentiated keratinocytes in the epidermis) can feature several pairings of types I and II keratins that, although expressed in a stage-specific fashion reflecting progression through differentiation, readily mix as proteins and/or subunits within individual 10-nm filaments (Coulombe and Omary 2002). The dual nature of keratins, their context-dependent regulation, and their propensity to coalesce into single polymers underlie an impressive potential for heterogeneity in filament composition, and filament structure and function (Kim and Coulombe 2007).

Genes encoding type I and type II keratins are each organized into compact clusters in mammalian genomes (Fig. 2B). In the human species, for instance, all type I keratin genes except the gene encoding K18 are clustered on the long arm of chromosome 17, whereas all the type II genes, along with KRT18, occur on the long arm of chromosome 12 (Hesse et al. 2004). There is an obvious relationship between sequence homology at the protein level and the arrangement of keratin genes in each of the type I and type II clusters (Fig. 2A,B), hinting at how gene-duplication events might have led to the current-day diversity within this prominent group of IFs. Furthermore, the clustered arrangement of keratin genes along with the suborganization of individual genes within the two clusters hint at the possibility of hierarchical regulation of their expression during embryogenesis and adult-tissue homeostasisan intriguing prospect that awaits further investigation.

\section{ASSEMBLY, PROPERTIES, AND ORGANIZATION OF KERATIN FILAMENTS IN EPITHELIAL CELLS}

The property of self-assembly typifies some cytoskeletal polymers. Equimolar mixtures of purified type I and II keratins readily copolymerize into long, uniform, and semiflexible 10-nm filaments with high efficiency (Fig. 1B) (Steinert et al. 1976). In vitro, the assembly reaction occurs in several steps, two of which are better understood biochemically-formation of coiled-coil heterodimers, in which the type I and II monomers interact in perfect register and parallel orientation, and then tetramers, in which the participating dimers interact along their lateral surfaces with an antiparallel orientation and staggered arrangement (Herrmann and Aebi 2004; Kim and Coulombe 2007). Keratin tetramers (K8-K18) can be isolated in relatively high yield from cultured cells and are believed to represent the predominant subunit precursor to filaments in living cells (Chou and Omary 1993). Keratin dimers and even tetramers can form under very harsh denaturing conditions, underscoring the unusual strength of the interactions between type I and type II keratins (Kim and Coulombe 2007). Tetramers interact along their lateral surfaces and in an end-to-end fashion as they come together to form 10-nm filaments (Herrmann and Aebi 2004; Kim and Coulombe 2007). Of note, keratin and other IFs are nonpolar fibrous cytoskeletal assemblies, demarcating them from F-actin and microtubules. Much remains to be learned about the fine structure of $10-\mathrm{nm}$ filaments, as well as their mode of assembly.

Likewise, relatively little is known about the process of keratin IF assembly as it occurs in the natural context of living cells. In epithelial cells in culture, keratins show an intriguing spatiotemporal cycle whereby small-sized precursors first appear at the cell periphery, near focal adhesions, and then elongate and integrate themselves into a network that undergoes continuous centripetal movement toward the centrally located nucleus (Windoffer et al. 2004). Keratin filament bundles then accumulate in the perinuclear region, where they can be stabilized, for example, by disulfide bonding (Feng and Coulombe 2015), or disassembled and turned over, allowing subunits to initiate new cycles at the cell periphery (Windoffer et al. 2011). Whether this paradigm applies to epithelial cells in their natural context in situ, and varies as a function of cell type, awaits further investigation.

Just like F-actin and microtubules, IFs are organized into intricate subcellular networks whose layout, in part, reflects their functional role, as well as that of the cell expressing them. Keratin IFs tend to be very abundant in surface-exposed stratified epithelia (Feng et al. 2013), where they typically form a pancytoplasmic network with attachments at the surface of the nucleus and sites of cellcell or cell-matrix adhesive structures spanning the outer membrane of the cell (Fig. 1D). In such settings, one of the primary roles of keratin filaments is to provide structural support (Coulombe et al. 1991b). In polarized simple epithelia, by contrast, keratins filaments are often concentrated at or near the apex and basolateral aspects of the cell (Fig. 1E) where they are positioned just beneath the F-actin-rich cortical cytoskeleton (Oriolo et al. 2007). In such tissues, one of the primary roles of keratin filaments is to resist chemical stresses and assist the cell in maintaining a polarized cytoarchitecture (Omary et al. 2009). As discussed below, plakin proteins mediate physical attachments among keratin IFs and other elements within the cell.

Whether functioning as individual 10-nm filaments or organized networks, keratin filaments can be highly dynamic entities (Miller et al. 1991; Windoffer et al. 2004). 
Keratin monomers appear very unstable in most biological settings, whereas keratin assemblies are inherently more stable but can undergo rapid turnover and/or be remodeled depending on the circumstances of the cell (e.g., undergoing mitosis, cell migration, or responding to stress) (Toivola et al. 2010). This property is a function of the interactions of keratins with other cellular proteins and, in particular, of their close regulation by site-specific phosphorylation and a variety of other posttranslational modifications (Snider and Omary 2014). These are described in Section 7 below.

From a biophysical and biomechanical perspective, keratin filaments behave like relatively weak gels when dispersed in solution (Ma et al. 2001). When cross-linked into networks, however, keratin filament suspensions adopt properties akin to solid materials and are able to withstand broad regimes of deformation and, yet, maintain their viscoelastic properties (Ma et al. 2001). Compared with suspensions of F-actin and microtubules at similar concentrations, IFs show a significantly greater ability to resist mechanical stress in vitro (Janmey et al. 1991), a property that has direct implications for their functional importance in vivo, as discussed in the next section.

\section{KERATIN FILAMENTS ARE ESSENTIAL FOR THE MAINTENANCE OF CELLULAR INTEGRITY IN THE FACE OF STRESS - A ROLE THAT IS DEFECTIVE IN EPITHELIAL FRAGILITY DISORDERS}

Consistent with their properties, abundance, and intracellular organization, it is no surprise that keratin filament assemblies significantly contribute to the mechanical resilience shown by several types of epithelial cells and tissues in vivo (Beil et al. 2003; Seltmann et al. 2013). Naturally occurring mutations that disrupt the structure of $10-\mathrm{nm}$ keratin filaments, their organization into intracellular networks within epithelial cells, and/or their regulation result in cellular fragility or altered responses to cell stress, and they are responsible as a cause or predisposition to several diseases affecting surface or internal epithelia and hair (Coulombe et al. 1991b; Omary et al. 2004; Szeverenyi et al. 2008; Coulombe and Lee 2012; Haines and Lane 2012; Toivola et al. 2015).

The first IF-based disorder discovered was epidermolysis bullosa simplex (EBS), a relatively rare condition (1:25,000 live births), inherited in an autosomal dominant fashion, that is caused by dominantly acting missense mutations in either the type I keratin 14 (K14) or the type II keratin 5 (K5) (Coulombe et al. 1991a; Coulombe and Lee 2012; Haines and Lane 2012). In individuals with EBS, the basal layer of the epidermis, in which $\mathrm{K} 5$ and K14 are prominently expressed, readily ruptures when the skin experiences frictional trauma, giving rise to characterizing bullous lesions or blisters (Fig. 3A). The notion that EBS could result from mutations in those specific keratin proteins was inferred from the phenotype shown by transgenic mice engineered to tissue-specifically express dominantly acting experimental mutations in K14 (Coulombe et al. 1991b,c; Vassar et al. 1991). Such mice showed traumainduced skin blistering similar to that seen in patients with EBS (Fig. 3B-E). This landmark set of findings spear-
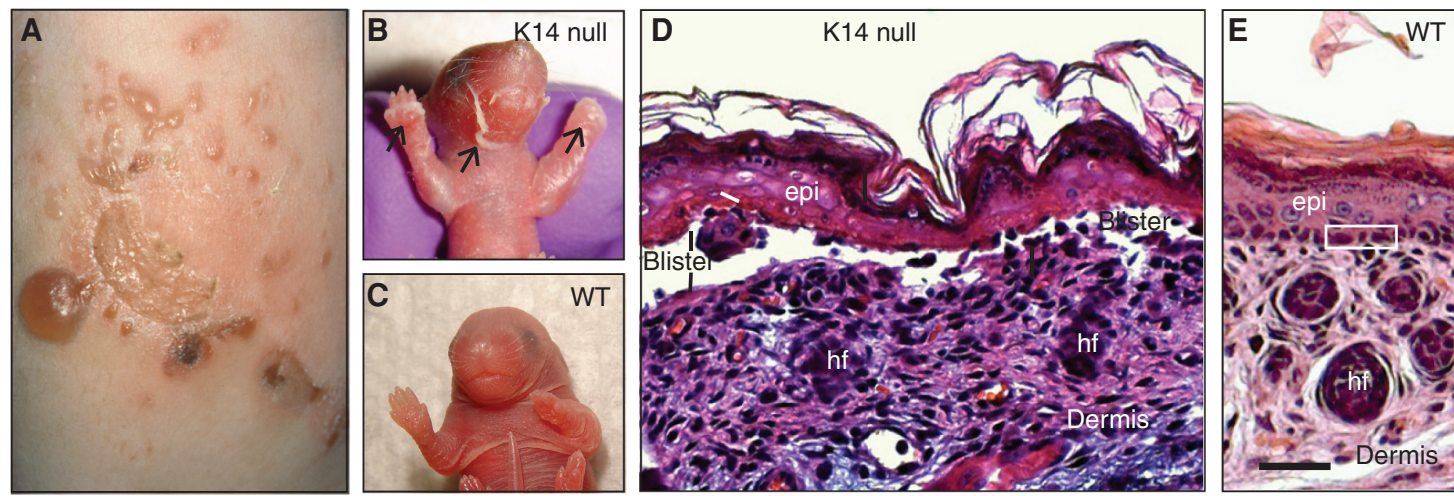

Figure 3. Loss of keratin K14 elicits epidermolysis bullosa simplex (EBS)-like features in mouse skin. (A) Leg skin in a patient suffering from the Dowling-Meara (severe) form of EBS. Characteristic of this severe variant of this disease, several skin blisters are grouped in a herpetiform fashion. $(B, C)$ Images of newborn mouse littermates, comparing K14-null and wild-type (WT) mice. The K14-null neonate shows massive skin blistering. The front paws and facial area are severely affected (arrows); by comparison, a WT littermate shows intact skin. $(D, E)$ Micrographs from hematoxylin/eosin-stained histological sections prepared from the front paws of 2-d-old K14-null and WT mice. Epidermal cleavage is obvious in the K14-null sample. Loss of epidermal integrity occurs through the basal layer of keratinocytes (labelled "blister")—-the defining characteristic of EBS. Three basal keratinocytes are boxed in E. epi, epidermis; hf, hair follicle. Scale bar, $100 \mu \mathrm{m}$. (A, Reprinted from Coulombe et al. 2013; B-E, adapted from Coulombe et al. 2009.) 
headed an explosion of discoveries in which mutations in other keratin and IF genes and proteins (usually either member of a given pair) were shown to underlie genetic conditions that bear resemblance to EBS at the cellular level. The identity of the tissue(s) affected by those mutations and, hence, the clinical presentation, is largely determined by the expression pattern of the mutated keratin gene (Omary et al. 2004). A compendium of keratin mutations and their associated diseases is available through the Intermediate Filament Database (www.interfil.org), which has important and helpful information regarding IF proteins and their associated diseases (Szeverenyi et al. 2008).

EBS and related conditions correspond to a loss of the structural support function, as evidenced by the phenotype arising in several keratin-null mutants in mice and also by the observation that disease-causing mutations alter the micromechanical properties of keratin filament assemblies in vitro and epithelial cells in culture (Ma et al. 2001; Werner et al. 2004). Moreover, the location and nature of a given mutation within a keratin sequence usually correlate with the extent to which it can disrupt filament assembly and/or organization and the severity of disease presentation in the afflicted individuals (Omary et al. 2004; Szeverenyi et al. 2008; Coulombe and Lee 2012). Hence, mutations that affect residues that are highly evolutionarily conserved (e.g., those located at the extremities of the central rod domain) tend to be associated with more severe disease presentation, whereas mutations affecting less wellconserved residues within the middle portion of the rod domain or in the nonhelical head and tail domains are associated with milder clinical presentations. Because of several complicating factors that include a broad spectrum of mutations, their dominant mode of action, and the inherently rapid rate of renewal occurring in most epithelial tissues, it has so far proven difficult to develop therapies to alleviate the symptoms associated with keratin disorders (Coulombe and Lee 2012). However, natural products or drugs that up-regulate normal keratin expression to compensate for the mutant keratin (Kerns et al. 2007) or enhance keratin binding to a stabilizing associated protein (Kwan et al. 2015) hold promise that similar approaches might provide novel drugs or identify drugs that can be repurposed to treat keratin and other IF-associated diseases for which there is no current direct treatment.

\section{EMERGENCE OF NONCANONICAL FUNCTIONS FOR KERATIN PROTEINS AND THEIR IMPLICATION IN DISEASE}

The diversity of keratins and exquisite regulation as a function of differentiation and biological context suggest that they partake in functions other than structural support.
Consistent with this, there are aspects of keratin-based diseases and transgenic mouse models in which keratin expression is manipulated that cannot be readily explained in terms of altered cellular mechanics. The first indication of a noncanonical role for keratins originated in the finding that expression of the K8-K18 pair protects epithelial cells against specific proapoptotic signals, both in culture and in vivo, in part, by attenuating the response to tumor necrosis factor $\alpha$ (TNF- $\alpha$ ) (Caulin et al. 2000) and Fas (Gilbert et al. 2001). A distinct mechanism through which K8$\mathrm{K} 18$, in particular, confers cytoprotection in the face of metabolic and/or proapoptotic stresses is to act as a sitespecific "phosphorylation sponge" for stress-activated kinases (Ku and Omary 2006). As keratins are very abundant proteins in epithelial cells, they are well poised to serve as effective "decoys" in the context of phosphorylation-dependent stress responses, ultimately allowing the cells and tissue to survive and adapt (Toivola et al. 2010). Keratinmediated cytoprotection plays a role in the setting of acute and chronic liver diseases, as shown in studies involving transgenic mouse models, along with the finding that missense alleles altering the coding sequence of K8 and K18 increase the risk for such conditions in the human population (Omary et al. 2009). Not only do keratins provide crucial protection from apoptosis, but type I (and not type II) keratins are major caspase substrates during apoptosis, with keratin caspase-generated fragments being used clinically as cancer and liver injury biomarkers (Omary et al. 2009). Inhibiting the caspase-mediated cleavage of K18 in transgenic mice by mutating the two aspartate cleavage sites prevents the $\mathrm{K} 8-\mathrm{K} 18$ pair from being able to undergo their typical reorganization during apoptosis and shunts hepatocytes toward necrosis (Weerasinghe et al. 2014). Hence, keratins are involved with apoptosis from several perspectives, including serving as caspase substrates and as biomarkers upon release from dying cells and as protectors from apoptotic injury by serving as phosphorylation sinks, although other mechanisms might also be involved in their cytoprotective role.

Keratins also participate in the regulation of epithelial tissue growth in at least three complementary ways. One is through regulation of progression through the cell cycle, which was originally observed in hepatocytes ( $\mathrm{Ku}$ et al. 2002) and oocytes (Margolis et al. 2006), and entails the phosphorylation-dependent interaction of K8-K18 with adaptor 14-3-3 proteins. A second way is through the regulation of protein synthesis, which has been observed in activated keratinocytes at the edge of skin wounds and in primary culture and involves an interaction between keratin 17 (K17) and a 14-3-3 protein (Kim et al. 2006; for review, see Cheng and Eriksson 2016). A role for K8-K18 in regulating protein synthesis and epithelial cell growth has also 
Types I and II Keratin Intermediate Filaments

been described in mid-stage developing mouse embryos and liver hepatocytes, probably through distinct mechanisms (Kellner and Coulombe 2009; Vijayaraj et al. 2009). Third, keratins participate in the expression or activation of powerful immune and inflammatory mediators that, once secreted, are able to stimulate epithelial tissue growth in both autocrine and paracrine (indirect) manners. Thus, the differentiation-related keratins $\mathrm{K} 1$ and $\mathrm{K} 16$ are important to keep the innate immune response in check in healthy epidermis, with $\mathrm{K} 1$ acting at the level of the inflammasome and cytokine activation (Roth et al. 2012), and K16 impacting the expression of danger-associated molecular patterns and skin barrier genes in response to external stresses (Lessard et al. 2013). As another example, K17 plays a significant role in the expression of several inflammatory and immune-response genes that are part of a growth-promoting signature in various types of cancers. This involves the interaction of K17 with the heterogeneous ribonucleoprotein hnRNP $\mathrm{K}$ and autoimmune regulator (AIRE), a transcriptional regulator functioning in the nucleus (Chung et al. 2015; Hobbs et al. 2015). Although much remains to be discovered and understood about the role of these and other keratins in epithelial growth control, it is already clear that this phenomenon is poised to impact several chronic inflammatory disorders, ranging from liver and bowel disorders to several types of skin conditions.

Finally, keratins also participate in the regulation of epithelial cell migration, a role that integrates their impact on cellular mechanics with their ability to regulate key signaling effectors. Evidence pointing to this role originally stems, yet again, from studies of disease processes such as cancer (Hendrix et al. 1996). The intracellular organization, dynamics, and phosphorylation-based regulation of keratin IFs are dramatically altered during cell migration (Chung et al. 2013). For instance, sphingophosphorylcholine-induced stimulation of epithelial tumor cell migration requires the phosphorylation-dependent collapse of keratin filaments around the nucleus and a softening of the cytoplasm (Beil et al. 2003). Skin wound re-epithelialization represents another setting in which keratin IFs become concentrated at the perinuclear region, correlating with an enhanced migration potential. Keratin 6 (K6), a woundinduced keratin, plays an important role in migrating keratinocytes by directly interacting with the Src tyrosine-protein kinase and regulating its ability to impact cell-matrix adhesion, a crucial determinant of cell migration (Rotty and Coulombe 2012). Yet another interesting example occurs in collectively migrating mesendoderm cells in Xenopus, whereby mechanical cues mediated by cadherin adhesion complexes induce polarization and directed migration in a plakoglobin- and keratin-dependent fashion (Weber et al. 2012).

\section{AN INTERPLAY BETWEEN KERATIN AND CYTOARCHITECTURE INVOLVES PROMINENT INTERACTIONS WITH THE PLAKIN FAMILY}

Plakin proteins play a significant role in mediating the physical attachment and functional integration of the association of IFs with F-actin and microtubules, with adhesion sites at the outer cell membrane, and with the surface of the nucleus in most cell types. The main plakin family proteins are plectin, BPAG1, desmoplakin, envoplakin, periplakin, epiplakin, and the microtubule-actin crosslinking factor 1. For many of these proteins, diversity is markedly enhanced through alternative splicing of precursor messenger RNAs (mRNAs) (Bouameur et al. 2014). In addition to a family-defining plakin domain, these proteins tend to be large and show a modular substructure, whereby several types of functional domains (e.g., actin-, IF-, and microtubule-binding domains) are arranged in a specific configuration to foster interactions between specific components. Accordingly, the functions of IFs and plakin family members are interdependent, and the consequences associated with plakin protein mutations in human and mouse show significant overlap with keratinbased diseases (Omary et al. 2004; Szeverenyi et al. 2008; Coulombe and Lee 2012; Bouameur et al. 2014). Likewise, there is emerging evidence that keratin IFs are important regulators of cell-matrix and cell-cell adhesion complexes, notably in the epidermis.

\section{KERATIN REGULATION BY POSTTRANSLATIONAL MODIFICATIONS}

Keratins undergo several posttranslational modifications (PTMs), including phosphorylation, O-linked glycosylation, ubiquitination, acetylation, SUMOylation, and transamidation (Fig. 4) (Snider and Omary 2014). Of these, phosphorylation has been studied more extensively than the others. Most of the known keratin phosphorylation sites are serine/threonine residues that are located in the head and tail domains of the keratins, although other stoichiometrically less abundant sites, including tyrosine residues within the rod domain, are becoming more readily appreciated, given the increasing sensitivity of mass spectrometry tools. One clear function of keratin phosphorylation is to enhance keratin solubility, which in turn triggers reorganization of the keratin filament network. Keratin reorganization then, through mechanisms that remain unclear, affords resistance to apoptosis (Snider and Omary 2014). In addition, and as mentioned above, one mechanism for cytoprotection by keratin phosphorylation is for the keratin to serve as a phosphate sponge or sink and thereby shunt undesirable phosphorylation of proapop- 


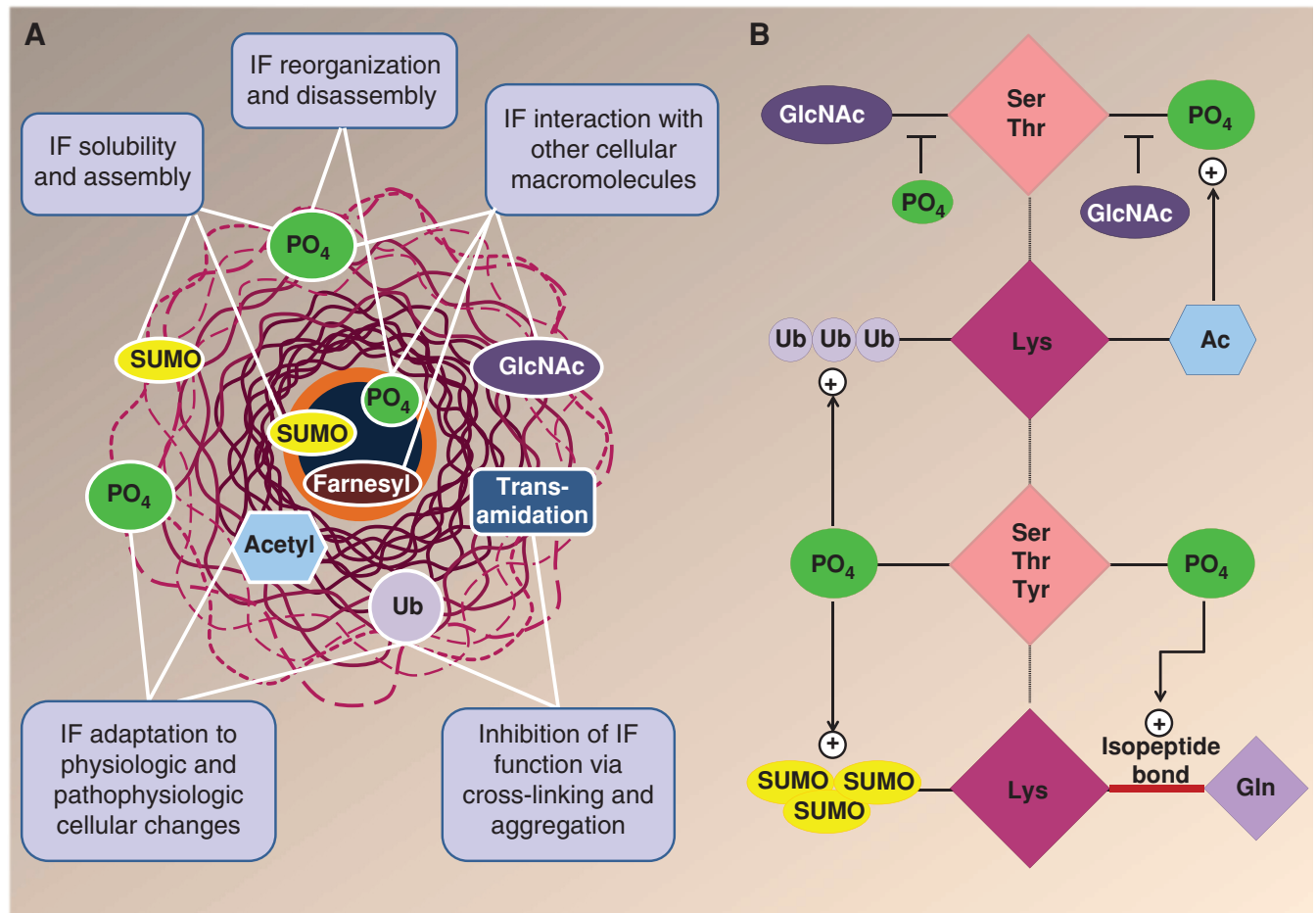

Figure 4. Posttranslational modifications (PTMs) of intermediate filament (IF) proteins. (A) IFs undergo multiple PTMs, including phosphorylation $\left(\mathrm{PO}_{4}\right)$, O-linked glycosylation (GlcNAc), ubiquitination (Ub), acetylation (acetyl), SUMOylation (SUMO), transamidation, and farnesylation (Snider and Omary 2014). Keratins also undergo all these modifications except for farnesylation, which specifically occurs in lamin IF proteins. Multiple functions are ascribed to IF/keratin PTMs, as highlighted in the panel text boxes. (B) Keratin PTMs target residues that can be modified by multiple PTMs. For example, Ser/Thr residues can be phosphorylated and glycosylated, whereas Lys residues can be modified by acetylation, ubiquitination, SUMOylation, and transamidation. There is also cross talk between different modifications, as exemplified by the effect of phosphorylation on several PTMs, and vice versa. The multiple combinations of modifications on a given keratin can provide a complex hierarchy of regulation. (Modified from Snider and Omary 2014.)

totic proteins by stress-activated kinases (Ku and Omary 2006). Hence, dynamic phosphorylation-dephosphorylation cycles coupled to keratin filament reorganization provide a reversible shock-absorber-type mechanism for cells to cope with stresses originating from the intracellular and extracellular environments.

Several keratin PTMs can target Ser/Thr or Lys residues (Fig. 4A,B). For example, glycosylation of keratins also occurs on serine residues, which provides for potential glycosylation-phosphorylation interregulation by these two PTMs, although the specific known modification sites appear to be different (Snider and Omary 2014). Keratin glycosylation appears to modulate the K8-K18 signaling scaffold, which includes kinases such as protein kinase C and Akt. For example, transgenic mice that express a K18 glycosylation-defective mutant become predisposed to liver injury in association with inactivation of Akt1 because of its hyperglycosylation and hypophosphorylation (Ku et al. 2010). Other potential keratin PTMs that involve a different type of cross talk occur at Lys residues and include SUMOy- lation, ubiquitination, acetylation, and transamidation (Fig. 4A,B). Keratin SUMOylation is barely detectable under basal conditions but is markedly enhanced during apoptosis, exposure to oxidative stress, phosphatase inhibition (typically in association with keratin hyperphosphorylation), and when the type I and type II keratin stoichiometry is different from the normal 1:1 ratio. The latter is the case in the context of certain forms of liver injury, when K8 transcription levels exceed those of K18 (Omary et al. 2009; Snider and Omary 2014). In terms of ubiquitination, this PTM is crucial for the turnover of keratins by the proteasome, which occurs during proteotoxic stress. This becomes relevant from a disease perspective because inhibition of the proteasome during chronic liver injury, coupled with activation of transglutaminase-2, results in the accumulation of transamidated and ubiquitinated cytoplasmic keratin pathological aggregates that are called Mallory-Denk bodies (Omary et al. 2009). There also appears to be cross talk between keratin lysine acetylation and keratin phosphorylation. For example, acetyla- 
tion of the highly conserved Lys207 residue of K8 decreases keratin solubility, increases keratin bundling in cell lines, and promotes $\mathrm{K} 8$ phosphorylation, presumably by a keratin conformational change that allows access of a keratin kinase (Snider and Omary 2014). Although not formally tested, it is possible that keratin lysine acetylation might also promote or blunt phosphorylation at adjacent Ser/ Thr residues, given that several kinase motifs include Lys residues at the $-1,-2$, or -3 position of a Ser/Thr kinase site.

\section{CONCLUSION}

Keratins are obligate heteropolymers that make up the two largest subgroups of the IF family of cytoskeletal proteins. They are regulated by several PTMs, along with a growing list of keratin-associated proteins. Keratins serve multiple cellular functions, with their most important shared role being to protect cells and tissues from injury. This function becomes magnified in the context of keratin mutations that cause or predispose to a wide range of epithelial disorders.

\section{ACKNOWLEDGMENTS}

We are grateful to members of the Coulombe and Omary laboratories for support. Our work is supported by National Institutes of Health (NIH) grants AR044232, AR042047, and CA160255 (to P.A.C.); and NIH grants DK47918 and DK52951, and Department of Veterans Affairs support (to M.B.O.). J.T.J. received support for NIH training grant T32 CA009110.

\section{REFERENCES}

* Reference is also in this collection.

Beil M, Micoulet A, von Wichert G, Paschke S, Walther P, Omary MB, Van Veldhoven PP, Gern U, Wolff-Hieber E, Eggermann J, et al. 2003. Sphingosylphosphorylcholine regulates keratin network architecture and visco-elastic properties of human cancer cells. Nat Cell Biol 5: 803-811.

Bouameur JE, Favre B, Borradori L. 2014. Plakins, a versatile family of cytolinkers: Roles in skin integrity and in human diseases. J Invest Dermatol 134: 885-894.

Caulin C, Ware CF, Magin TM, Oshima RG. 2000. Keratin-dependent, epithelial resistance to tumor necrosis factor-induced apoptosis. J Cell Biol 149: 17-22.

* Cheng F, Eriksson JE. 2016. Intermediate filaments and the regulation of cell motility during regeneration and wound healing. Cold Spring Harb Perspect Biol doi: 101101/cshperspect.a022046.

Chou CF, Omary MB. 1993. Mitotic arrest-associated enhancement of O-linked glycosylation and phosphorylation of human keratins 8 and 18. J Biol Chem 268: 4465-4472.

Chung BM, Rotty JD, Coulombe PA. 2013. Networking galore: Intermediate filaments and cell migration. Current Opin Cell Biol 25: 600-612.

Chung BM, Arutyunov A, Ilagan E, Yao N, Wills-Karp M, Coulombe PA. 2015. Regulation of C-X-C chemokine gene expression by keratin 17 and hnRNP K in skin tumor keratinocytes. J Cell Biol 208: 613-627.
Coulombe PA, Omary MB. 2002. "Hard" and "soft" principles defining the structure, function and regulation of keratin intermediate filaments. Curr Opin Cell Biol 14: 110-122.

Coulombe PA, Lee CH. 2012. Defining keratin protein function in skin epithelia: Epidermolysis bullosa simplex and its aftermath. J Invest Dermatol 132: 763-775.

Coulombe PA, Hutton ME, Letai A, Hebert A, Paller AS, Fuchs E. 1991a. Point mutations in human keratin 14 genes of epidermolysis bullosa simplex patients: Genetic and functional analyses. Cell 66: 1301-1311.

Coulombe PA, Hutton ME, Vassar R, Fuchs E. 1991b. A function for keratins and a common thread among different types of epidermolysis bullosa simplex diseases. J Cell Biol 115: 1661-1674.

Coulombe PA, Hutton ME, Vassar R, Fuchs E. 1991c. A function for keratins and a common thread among different types of epidermolysis bullosa simplex diseases. J Cell Biol 115: 1661-1674.

Coulombe PA, Kerns ML, Fuchs E. 2009. Epidermolysis bullosa simplex: A paradigm for disorders of tissue fragility. J Clin Invest 119: 17841793.

Coulombe PA, Bernot KM, Lee CH. 2013. Keratins and the skin. In Encyclopedia of biological chemistry, 2nd ed. (ed. Lane MD, Lennarz WJ), pp. 665-671. Elsevier Science, New York.

Feng X, Coulombe PA. 2015. A role for disulfide bonding in keratin intermediate filament organization and dynamics in skin keratinocytes. J Cell Biol 209: 59-72.

Feng X, Zhang H, Margolick JB, Coulombe PA. 2013. Keratin intracellular concentration revisited: Implications for keratin function in surface epithelia. J Invest Dermatol 133: 850-853.

Fuchs E. 1995. Keratins and the skin. Annu Rev Cell Dev Biol 11: 123-153.

Gilbert S, Loranger A, Daigle N, Marceau N. 2001. Simple epithelium keratins 8 and 18 provide resistance to Fas-mediated apoptosis. The protection occurs through a receptor-targeting modulation. J Cell Biol 154: $763-774$.

Haines RL, Lane EB. 2012. Keratins and disease at a glance. J Cell Sci 125: 3923-3928.

Hendrix MJ, Seftor EA, Chu YW, Trevor KT, Seftor RE. 1996. Role of intermediate filaments in migration, invasion and metastasis. Cancer Metastasis Rev 15: 507-525.

Herrmann H, Aebi U. 2004. Intermediate filaments: Molecular structure, assembly mechanism, and integration into functionally distinct intracellular scaffolds. Annu Rev Biochem 73: 749-789.

* Herrmann H, Aebi U. 2016. Intermediate filaments: Structure and assembly. Cold Spring Harb Perspect Biol 8: a018242.

Hesse M, Zimek A, Weber K, Magin TM. 2004. Comprehensive analysis of keratin gene clusters in humans and rodents. Eur J Cell Biol 83: $19-26$.

Hobbs RP, DePianto DJ, Jacob JT, Han MC, Chung BM, Batazzi AS, Poll BG, Guo Y, Han J, Ong S, et al. 2015. Keratin-dependent regulation of Aire and gene expression in skin tumor keratinocytes. Nat Genet 47: 933-938.

Ishikawa H, Bischoff R, Holtzer H. 1968. Mitosis and intermediate-sized filaments in developing skeletal muscle. J Cell Biol 38: 538-555.

Janmey PA, Euteneuer U, Traub P, Schliwa M. 1991. Viscoelastic properties of vimentin compared with other filamentous biopolymer networks. J Cell Biol 113: 155-160.

Kellner JC, Coulombe PA. 2009. Keratins and protein synthesis: The plot thickens. J Cell Biol 187: 157-159.

Kerns ML, DePianto D, Dinkova-Kostova AT, Talalay P, Coulombe PA. 2007. Reprogramming of keratin biosynthesis by sulforaphane restores skin integrity in epidermolysis bullosa simplex. Proc Natl Acad Sci 104: 14460-14465.

Kim S, Coulombe PA. 2007. Intermediate filament scaffolds fulfill mechanical, organizational, and signaling functions in the cytoplasm. Genes Dev 21: 1581-1597.

Kim S, Wong P, Coulombe PA. 2006. A keratin cytoskeletal protein regulates protein synthesis and epithelial cell growth. Nature 441: 362365. 


\section{J.T. Jacob et al.}

Ku NO, Omary MB. 2006. A disease- and phosphorylation-related nonmechanical function for keratin 8. J Cell Biol 174: 115-125.

Ku NO, Michie S, Resurreccion EZ, Broome RL, Omary MB. 2002. Keratin binding to 14-3-3 proteins modulates keratin filaments and hepatocyte mitotic progression. Proc Natl Acad Sci 99: 4373-4378.

Ku NO, Toivola DM, Strnad P, Omary MB. 2010. Cytoskeletal keratin glycosylation protects epithelial tissue from injury. Nat Cell Biol 12: 876-885.

Kwan R, Chen L, Looi K, Tao GZ, Weerasinghe SV, Snider NT, Conti MA, Adelstein RS, Xie Q, Omary MB. 2015. PKC412 normalizes mutationrelated keratin filament disruption and hepatic injury in mice by promoting keratin-myosin binding. Hepatology 62: 1858-1869.

Lessard JC, Pina-Paz S, Rotty JD, Hickerson RP, Kaspar RL, Balmain A, Coulombe PA. 2013. Keratin 16 regulates innate immunity in response to epidermal barrier breach. Proc Natl Acad Sci 110: 19537-19542.

Ma L, Yamada S, Wirtz D, Coulombe PA. 2001. A "hot-spot" mutation alters the mechanical properties of keratin filament networks. Nat Cell Biol 3: 503-506.

Margolis SS, Perry JA, Forester CM, Nutt LK, Guo Y, Jardim MJ, Thomenius MJ, Freel CD, Darbandi R, Ahn JH, et al. 2006. Role for the PP2A/B56 phosphatase in regulating 14-3-3 release from Cdc25 to control mitosis. Cell 127: 759-773.

Miller RK, Vikstrom K, Goldman RD. 1991. Keratin incorporation into intermediate filament networks is a rapid process. J Cell Biol 113: 843855

Moll R, Franke WW, Schiller DL, Geiger B, Krepler R. 1982. The catalog of human cytokeratins: Patterns of expression in normal epithelia, tumors and cultured cells. Cell 31: 11-24.

Moll R, Divo M, Langbein L. 2008. The human keratins: Biology and pathology. Histochem Cell Biol 129: 705-733.

Norlén L, Al-Amoudi A. 2004. Stratum corneum keratin structure, function, and formation: The cubic rod-packing and membrane templating model. J Invest Dermatol 123: 715-732.

Omary MB, Coulombe PA, McLean WHI. 2004. Intermediate filament proteins and their associated diseases. $N$ Engl J Med 351: 2087-2100.

Omary MB, Ku NO, Strnad P, Hanada S. 2009. Toward unraveling the complexity of simple epithelial keratins in human disease. J Clin Invest 119: $1794-1805$

Oriolo AS, Wald FA, Ramsauer VP, Salas PJ. 2007. Intermediate filaments: A role in epithelial polarity. Exp Cell Res 313: 2255-2264.

Roth W, Kumar V, Beer HD, Richter M, Wohlenberg C, Reuter U, Thiering S, Staratschek-Jox A, Hofmann A, Kreusch F, et al. 2012. Keratin 1 maintains skin integrity and participates in an inflammatory network in skin through interleukin-18. J Cell Sci 125: 5269-5279.

Rotty JD, Coulombe PA. 2012. A wound-induced keratin inhibits Src activity during keratinocyte migration and tissue repair. J Cell Biol 197: $381-389$.

Schweizer J, Bowden PE, Coulombe PA, Langbein L, Lane EB, Magin TM, Maltais L, Omary MB, Parry DA, Rogers MA, et al. 2006. New con- sensus nomenclature for mammalian keratins. J Cell Biol 174: 169_ 174.

Seltmann K, Fritsch AW, Kas JA, Magin TM. 2013. Keratins significantly contribute to cell stiffness and impact invasive behavior. Proc Natl Acad Sci 110: 18507-18512.

Snider NT, Omary MB. 2014. Post-translational modifications of intermediate filament proteins: Mechanisms and functions. Nat Rev Mol Cell Biol 15: 163-177.

Steinert PM, Idler WW, Zimmerman SB. 1976. Self-assembly of bovine epidermal keratin filaments in vitro. J Mol Biol 108: 547-567.

Strnad P, Usachov V, Debes C, Grater F, Parry DA, Omary MB. 2011. Unique amino acid signatures that are evolutionarily conserved distinguish simple-type, epidermal and hair keratins. J Cell Sci 124: $4221-4232$

Sun TT, Eichner R, Nelson WG, Tseng SC, Weiss RA, Jarvinen M, Woodcock-Mitchell J. 1983. Keratin classes: Molecular markers for different types of epithelial differentiation. J Invest Dermatol 81: 109s-115s.

Szeverenyi I, Cassidy AJ, Chung CW, Lee BT, Common JE, Ogg SC, Chen H, Sim SY, Goh WL, Ng KW, et al. 2008. The Human Intermediate Filament Database: Comprehensive information on a gene family involved in many human diseases. Hum Mutat 29: 351-360.

Toivola DM, Strnad P, Habtezion A, Omary MB. 2010. Intermediate filaments take the heat as stress proteins. Trends Cell Biol 20: 79-91.

Toivola DM, Boor P, Alam C, Strnad P. 2015. Keratins in health and disease. Curr Opin Cell Biol 32: 73-81.

Vassar R, Coulombe PA, Degenstein L, Albers K, Fuchs E. 1991. Mutant keratin expression in transgenic mice causes marked abnormalities resembling a human genetic skin disease. Cell 64: 365-380.

Vijayaraj P, Kröger C, Reuter U, Windoffer R, Leube RE, Magin TM. 2009. Keratins regulate protein biosynthesis through localization of GLUT1 and -3 upstream of AMP kinase and Raptor. J Cell Biol 187: 175-184.

Weber GF, Bjerke MA, DeSimone DW. 2012. A mechanoresponsive cadherin-keratin complex directs polarized protrusive behavior and collective cell migration. Dev Cell 22: 104-115.

Weerasinghe SV, Ku NO, Altshuler PJ, Kwan R, Omary MB. 2014. Mutation of caspase-digestion sites in keratin 18 interferes with filament reorganization, and predisposes to hepatocyte necrosis and loss of membrane integrity. J Cell Sci 127: 1464-1475.

Werner NS, Windoffer R, Strnad P, Grund C, Leube RE, Magin TM. 2004. Epidermolysis bullosa simplex-type mutations alter the dynamics of the keratin cytoskeleton and reveal a contribution of actin to the transport of keratin subunits. Mol Biol Cell 15: 990-1002.

Windoffer R, Woll S, Strnad P, Leube RE. 2004. Identification of novel principles of keratin filament network turnover in living cells. Mol Biol Cell 15: 2436-2448.

Windoffer R, Beil M, Magin TM, Leube RE. 2011. Cytoskeleton in motion: The dynamics of keratin intermediate filaments in epithelia. J Cell Biol 194: 669-678. 


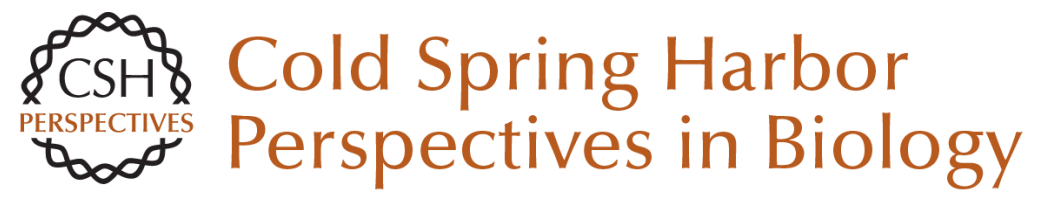

\section{Types I and II Keratin Intermediate Filaments}

Justin T. Jacob, Pierre A. Coulombe, Raymond Kwan and M. Bishr Omary

Cold Spring Harb Perspect Biol 2018; doi: 10.1101/cshperspect.a018275

Subject Collection The Cytoskeleton

Microtubules and Microtubule-Associated

Proteins

Holly V. Goodson and Erin M. Jonasson

Motor Proteins

H. Lee Sweeney and Erika L.F. Holzbaur

Myosin-Driven Intracellular Transport Margaret A. Titus

The Actin Cytoskeleton and Actin-Based Motility Tatyana Svitkina

\section{Mechanical Properties of the Cytoskeleton and Cells \\ Adrian F. Pegoraro, Paul Janmey and David A.} Weitz

Intermediate Filaments and the Regulation of Cell Motility during Regeneration and Wound Healing Fang Cheng and John E. Eriksson

Intermediate Filaments and the Plasma Membrane Jonathan C.R. Jones, Chen Yuan Kam, Robert M. Harmon, et al.

Intracellular Motility of Intermediate Filaments Rudolf E. Leube, Marcin Moch and Reinhard Windoffer

\section{Overview of the Cytoskeleton from an}

Evolutionary Perspective

Thomas D. Pollard and Robert D. Goldman

Types I and II Keratin Intermediate Filaments Justin T. Jacob, Pierre A. Coulombe, Raymond Kwan, et al.

Muscle Contraction

H. Lee Sweeney and David W. Hammers

Type III Intermediate Filaments Desmin, Glial

Fibrillary Acidic Protein (GFAP), Vimentin, and

Peripherin

Elly M. Hol and Yassemi Capetanaki

Cytokinesis in Metazoa and Fungi Michael Glotzer

Ciliary Motility: Regulation of Axonemal Dynein Motors

Rasagnya Viswanadha, Winfield S. Sale and Mary

E. Porter

Actin-Based Adhesion Modules Mediate Cell Interactions with the Extracellular Matrix and Neighboring Cells

Alexia I. Bachir, Alan Rick Horwitz, W. James Nelson, et al.

Microtubule-Based Transport and the Distribution,

Tethering, and Organization of Organelles Kari Barlan and Vladimir I. Gelfand

For additional articles in this collection, see http://cshperspectives.cshlp.org/cgi/collection/

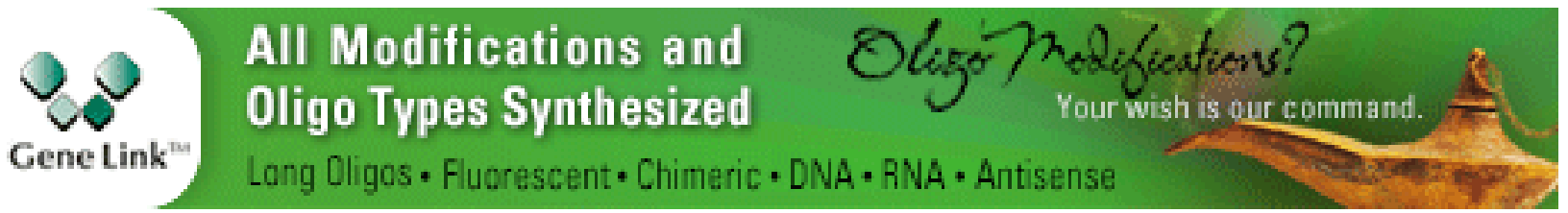

Copyright @ 2018 Cold Spring Harbor Laboratory Press; all rights reserved 
For additional articles in this collection, see http://cshperspectives.cshlp.org/cgi/collection/

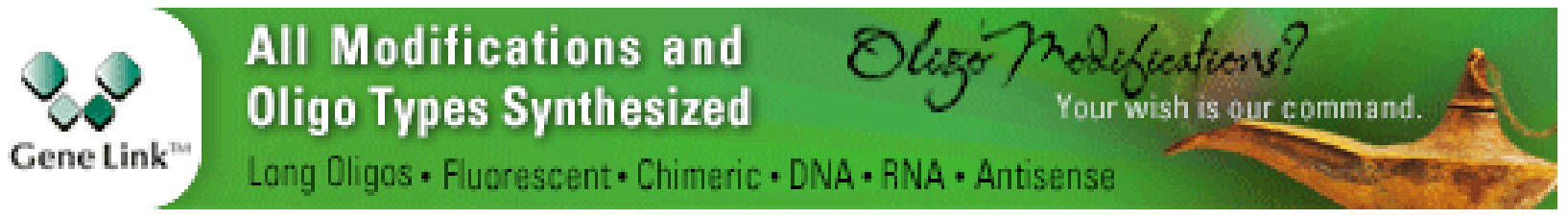

Copyright @ 2018 Cold Spring Harbor Laboratory Press; all rights reserved 